\title{
Innovative Education and Training in high power laser plasmas (PowerLaPs) for plasma physics, high power laser-matter interactions and high energy density physics - theory and experiments
}

John Pasley ${ }^{1}$, Georgia Andrianaki ${ }^{2}$, Andreas Baroutsos ${ }^{2}$, Dimitri Batani ${ }^{3}$, Emmanouil P. Benis ${ }^{4}$, Marco Borghesi $^{5}$, Eugene Clark ${ }^{2}$, Donna Cook ${ }^{1}$, Emmanuel D'Humieres ${ }^{3}$, Vasilios Dimitriou ${ }^{2}$, Brendan Dromey ${ }^{5}$, Michael Ehret ${ }^{3}$, Ioannis Fitilis ${ }^{2}$, Anastasios Grigoriadis ${ }^{2}$, Satya $\mathrm{Kar}^{5}$, Evaggelos Kaselouris ${ }^{2}$, Ondrej $\mathrm{Klimo}^{6}$, Michel Koenig ${ }^{7}$, Kyriaki Kosma ${ }^{2}$, George Koundourakis ${ }^{2}$, Milan Kucharik ${ }^{6}$, Aveen Lavery ${ }^{5}$, Jiri Limpouch ${ }^{6}$, Yannis Orphanos ${ }^{2}$, Nektarios A. Papadogiannis ${ }^{2}$, Stelios Petrakis ${ }^{2}$, Dave Riley ${ }^{5}$, Maria Serena Rivetta ${ }^{8}$, Laura Tejada Pascual ${ }^{3}$, João Jorge Santos ${ }^{3}$, Alexandros Skoulakis ${ }^{2}$, Ioannis Tazes ${ }^{2}$, Vladimir Tikhonchuk ${ }^{3}$, Jocelain Trela ${ }^{3}$, Calliope Tsitou ${ }^{2}$, Luca Volpe ${ }^{9,10}$, Steven White ${ }^{5}$, Mark Yeung ${ }^{5}$, and Michael Tatarakis ${ }^{2}$

${ }^{1}$ York Plasma Institute, University of York, Heslington, York YO10 5DQ, UK

${ }^{2}$ Technological Educational Institute of Crete, School of Applied Sciences, Centre for Plasma Physics and Lasers-CPPL, 74100 Rethymnon, 73133 Chania, Crete, Greece

${ }^{3}$ Université de Bordeaux, CNRS, CEA, CELIA (Centre Lasers Intenses et Applications), UMR 5107, F-33405 Talence, France

${ }^{4}$ Department of Physics, University of Ioannina, 45110 Ioannina, Greece

${ }^{5}$ Centre for Plasma Physics, School of Mathematics and Physics, Queen's University Belfast, Belfast BT7 1NN, UK

${ }^{6}$ Faculty of Nuclear Sciences and Physical Engineering, Czech Technical University in Prague, Brehova 7, 115 19, Prague 1, Czech Republic

${ }^{7}$ LULI - CNRS, Ecole Polytechnique, CEA : Université Paris-Saclay; UPMC Univ Paris 06 : Sorbonne Universités, F-91128 Palaiseau cedex, France

${ }^{8}$ University Institute for Educational Sciences, University of Salamanca, 37008 Salamanca, Spain

${ }^{9}$ CLPU Laser-Plasma Chair, University of Salamanca, 37008 Salamanca, Spain

${ }^{10}$ Centro de Láseres Pulsados (CLPU), Edificio M5. Parque Cientfico. C/ Adaja, 8. 37185 Villamayor, Salamanca, Spain

(Received 20 December 2018; accepted 21 January 2019)

\begin{abstract}
The Erasmus Plus programme 'Innovative Education and Training in high power laser plasmas', otherwise known as PowerLaPs, is described. The PowerLaPs programme employs an innovative paradigm in that it is a multi-centre programme where teaching takes place in five separate institutes with a range of different aims and styles of delivery. The 'in class' time is limited to four weeks a year, and the programme spans two years. PowerLaPs aims to train students from across Europe in theoretical, applied and laboratory skills relevant to the pursuit of research in laserplasma interaction physics and inertial confinement fusion (ICF). Lectures are intermingled with laboratory sessions and continuous assessment activities. The programme, which is led by workers from the Technological Educational Institute (TEI) of Crete, and supported by co-workers from the Queen's University Belfast, the University of Bordeaux, the Czech Technical University in Prague, Ecole Polytechnique, the University of Ioannina, the University of Salamanca and the University of York, has just completed its first year. Thus far three Learning Teaching Training (LTT) activities have been held, at the Queen's University Belfast, the University of Bordeaux and the Centre for Plasma Physics and Lasers (CPPL) of TEI Crete. The last of these was a two-week long Intensive Programme (IP), while the activities at the other two universities were each five days in length. Thus far work has concentrated upon training in both theoretical and experimental work in plasma physics, high power laser-matter interactions and high energy density physics. The nature of the programme will be described in detail and some metrics relating to the activities carried out to date will be presented.
\end{abstract}

Keywords: higher education; inertial confinement fusion; laser-plasma interactions; post-graduate education

Correspondence to: J. Pasley, York Plasma Institute, University of York,

York YO10 5DQ, UK. Email: john.pasley@york.ac.uk 


\section{Introduction}

'Innovative Education and Training in high power laser plasmas' - PowerLaPs ${ }^{[1]}$ is an Erasmus Plus - KA2 ${ }^{[2]}$ programme that aims to assist and enhance the studies of final year Bachelor students, Masters students and $\mathrm{PhD}$ students; improving their employability skills in the scientific area of plasma physics and high power lasers. The programme focuses upon four thematic priority areas: (i) plasma physics, (ii) high power laser-matter interactions and high energy density physics in theory and practice, (iii) computational modelling and simulations in laser-matter interactions, and (iv) plasma diagnostics. None of these areas are commonly included in university curricula, though some of the individual universities involved in the PowerLaPs programme do offer training in these areas separately ${ }^{[3-6]}$. PowerLaPs is innovative for a training course in the physical sciences in that it is an intermittent educational experience, in which the students attend classes for only four weeks a year, yet the training is split across multiple institutes, and at each centre the teaching is delivered in a range of different ways including formal lectures, experimental laboratory sessions, computational laboratory sessions, and continuous assessment (the last of these activities also continues outside the period of the workshops).

The main aim of the programme is to challenge the student participants to expand their knowledge and scientific skills in areas that are relevant to the current state of the art research in the field of laser-plasma interactions and inertial fusion energy ${ }^{[7]}$. This area of expertise is relevant to a range of facilities that are either built, or being built both in Europe and internationally, for instance the National Ignition Facility $(\mathrm{NIF})^{[8]}$, the Extreme Light Infrastructure (ELI) ${ }^{[9]}$, the CILEX-APOLLON ${ }^{[10]}$ and the European X-ray Free Electron Laser (XFEL) $)^{[11]}$.

Student attendees gain additional soft skills and expand their research networks by interaction with both students and academics in a range of different settings from quite formal lecture-style teaching through to social settings. This encourages cross-border cooperation and enhances the professional skills of the attendees.

Throughout the programme there are a series of assessment exercises which test the students grasp of the material that has been delivered. These take the form of both openbook written exams carried out in a controlled environment (the results of which are discussed below) as well as longer course-work exercises which are completed in the students' own time outside of the lectures and laboratories.

One interesting feature of the programme is that, although the Learning Teaching Training (LTT) activities and two Intensive Programmes (IPs) that form the basis of the PowerLaPs are constructed as a single educational course, students are not required to attend all of the sessions. Students receive individual certification for attendance at each of the
LTTs/IPs, and can opt to attend from one to all of the events. For this reason, the individual taught modules have to be carefully designed to enable the participation of students who have not necessarily attended previous workshops. The results of the open-book examinations performed at the end of each of the LTTs/IP to date are assessed below to determine whether failure to attend earlier training events is detrimental to student performance.

The material developed as part of the PowerLaPs programme will be made available worldwide through the PowerLaPs website, which is developed partially in parallel to the evolution of the programme ${ }^{[1]}$. At present, and until the completion of the course, these resources are only available to students from the institutes participating in the programme via the e-Class platform hosted by the Technological Educational Institute (TEI) of Crete.

\section{Subject areas}

The different topics covered by the PowerLaPs programme are divided into four areas of specialization labelled $\mathrm{O} 1, \mathrm{O} 2$, $\mathrm{O} 3$ and $\mathrm{O} 4$. Each of these is described below.

\subsection{O1 Plasma physics - theory and experiments}

O1 is the theoretical and experimental part of the Plasma Physics thematic priority educational material. The LTT in this area took place at the University of Bordeaux in January 2018, with further education in this area taking place at the first IP that was held at TEI Crete in July 2018. The LTT-C1 at Bordeaux included the following topics delivered in a lecture-style format: (1) introduction to laser safety, (2) introduction and classification of plasmas, (3) movement of charged particles in electric and magnetic fields, (4) hydrodynamic (two-fluid) description of a plasma, (5) kinetic description of waves and instabilities in plasmas, and (6) radiative properties of plasmas; and in addition the following laboratory sessions: (1) laser discharge at the surface of a solid target and propagation of a deflagration wave in air (experimental), (2) two-stream instability and plasma expansion in vacuum exercises with the SMILEI code (computational), and (3) plasma radiation calculations with the FLYCHK code (computational). Please also see the following subsection ' $\mathrm{O} 2$ ' where the materials delivered at the IP are described for both $\mathrm{O} 1$ and $\mathrm{O} 2$.

\subsection{O2 High power laser-matter interactions/high energy density physics - theory and experiments}

$\mathrm{O} 2$ is the theoretical and experimental part of the high power laser-matter interactions/high energy density physics material. The LTT in this area took place at Queen's University Belfast in March 2018, with further education in this 
area taking place at the first IP that was held at TEI Crete in July 2018. The LTT in Belfast included the following topics, delivered in lecture-style format: (1) fundamentals of laser-pulse production, (2) optical cavity design, (3) ultrafast laser pulses, (4) attosecond pulse trains, (5) high harmonic generation, (6) warm dense matter, and (7) laser-driven ion acceleration; and in addition the following laboratory sessions: (1) laser alignment, expansion and beam collimation (experimental), (2) measuring coherence length and time (experimental), and (3) X-ray absorption spectroscopy (experimental).

The first IP, held at Centre for Plasma Physics and Lasers (CPPL) in Crete, spanned both $\mathrm{O} 1$ and $\mathrm{O} 2$. The lectures and laboratories held at the IP that are relevant to these two topics are therefore listed together. The following topics were delivered in a lecture-style format: (1) introduction to and classification of plasmas and particle motion in a plasma, (2) ultrafast laser-matter interactions, (3) basics of laser-atom interactions and atomic processes in plasma, (4) coherent XUV sources, (5) simulations and numerical modelling in plasma physics and laser-matter interactions, (6) from perfect gas to QDIM: applications to planetary physics, (7) hydrodynamic instabilities and implications in inertial confinement fusion (ICF) and astrophysics, (8) particle acceleration using lasers in plasmas, (9) warm dense matter, (10) high power lasers, (11) laser-driven proton sources and applications, (12) energy transport by lasergenerated fast electron beams in dense matter, (13) principles of indirect drive ICF physics, (14) shock waves and implosion hydrodynamics; and in addition the following laboratory sessions: (1) laser-matter interactions (experimental), (2) plasma focus (experimental), (3) plasma pinch (experimental), (4) particle-in-cell simulations of laser-plasma interaction (computational), (5) FEM simulations of laserplasma interaction (computational), and (6) magnetohydrodynamics simulations of plasma pinch (computational).

\subsection{O3 Computational modelling and simulations in laser-matter interactions}

The computational modelling and simulations in lasermatter interactions material will first be delivered at the Prague Technical University in early 2019, followed by further training at the second IP in summer 2019 at TEI Crete. The material delivered will be described in more detail in a follow-up paper.

\subsection{O4 Laser plasma diagnostics - theory and experiments}

The laser plasma diagnostics related educational material will first be delivered at the University of Salamanca in early 2019 , followed by further training at the second IP in summer 2019 at TEI Crete. The material delivered will be described in more detail in a follow-up paper.

\section{Participation and usage statistics}

Thus far the resources offered by the PowerLaPs programme have been taken advantage of well by all of the participating institutes. More detailed figures are provided below both for LTT attendance and e-Class platform usage.

\subsection{LTT attendance}

All three of the LTTs conducted to date have been well attended. Nonlocal institutes received funding from the programme to send three students to each of the LTTs/IPs meaning that the number of visiting students that can be funded to each activity is 21 , with the home institute sometimes having a larger number of students participate. The LTT held in January 2018 at the University of Bordeaux hosted 22 students (of which 20 completed the assessment activity for this LTT). The LTT held in March 2018 at Queen's University Belfast hosted 24 students. The first IP held in July 2018 hosted 29 students.

\section{2. e-Class platform usage}

The e-Class platform (which is currently only accessible to teachers, tutors and students from the partner universities) has been used by all of the students who have participated in the programme in addition to some others from the participating institutes. The material related to $\mathrm{O} 1$ has thus far been visited by 78 participants, of which 11 were academics and the remaining 67 students. The total number of visits made to the site by these users was 389 . The educational material related to $\mathrm{O} 2$ has received 76 visitors of whom 64 were students and the remainder academics. The total number of site visits by these users was 425 . It is noteworthy that the total number of students to be formally involved in at least one of the LTTs/IP of the PowerLaPs programme to date is 48 . This suggests that at least $25 \%$ of the students who have used the e-Class platform were using it independently of formal participation in the PowerLaPs programme.

\section{Student performance}

In the Bordeaux LTT the average mark attained across the cohort of 21 student attendees was $61.00 \%$ with a standard deviation of 16.57. In the Belfast LTT the average mark attained by the 24 student attendees was $77.25 \%$ with a standard deviation of 7.79. In the first IP in Crete the average mark among the 29 student attendees was $63.86 \%$ with a standard deviation of 5.57. The spread of marks for each of the three events is shown separately in Figure 1.

The change in standard deviation about the mean between the three schools is quite striking, and is put down to the 
fact that the assessments were designed and graded independently by academics at the host institutes of the respective LTTs/IP. There has been no formal effort to standardize the assessment practices between the three events, and this is perhaps most evident in the dramatic change in the standard deviation. The mean scores also show some variation, with the mean scores from the Belfast school being significantly higher than for the other two events.

There is no requirement placed upon students to attend all of the training LTTs, and while this is preferred, it clearly cannot be made a firm requirement since students have many competing demands placed upon their time and they may have good reason for not being able to attend all of the events. Furthermore, it is the case that sometimes the home institution for a given LTT may wish more students to attend than would be possible for one of the nonlocal LTTs. For this reason individual training courses are designed to be capable of being taken on a stand-alone basis. To examine the extent to which this ambition has been successfully achieved it is interesting to compare student performance on the IP between students who have attended all of the previous LTTs vs those whose first formal contact with the programme was at the first Cretan IP. The mean mark attained by students who attended all three of the LTTs on the first IP was $61.71 \%(N=7)$. The mean mark attained by students who attended one LTT prior to the first IP, on the exam of the first IP was $65.20 \%(N=10)$ and the mean mark attained on the exam of the first IP, for students whose first experience of the programme was at that IP was $64.00 \%(N=12)$. The fluctuation in the mean between these three groups is not significant, and it suggests that the instructors were successful in designing their courses so as to be comprehensible to a student who had not attended one of the previous LTTs of the PowerLaPs programme.

In any of the three LTTs/IP only one student attained a failing grade (assessment score less than 40\%). This student only participated in the first of the three events, held at Bordeaux, and received a score of $21 \%$. Given that the performance of this student indicates they were either not engaged with the programme, or were otherwise affected by some unusual situation, we have repeated the analysis having removed this student from the calculation. If this student is removed from the calculations of the mean and standard deviation then the reported numbers for the Bordeaux LTT change to $66.32 \%$ and 13.47 , respectively. The original marks are reported in Figure 1. To preserve anonymity, further information concerning the student who failed is not presented here.

\section{Dissemination}

PowerLaPs outputs are being continuously assembled into an e-Class platform that is open to partner institutions that
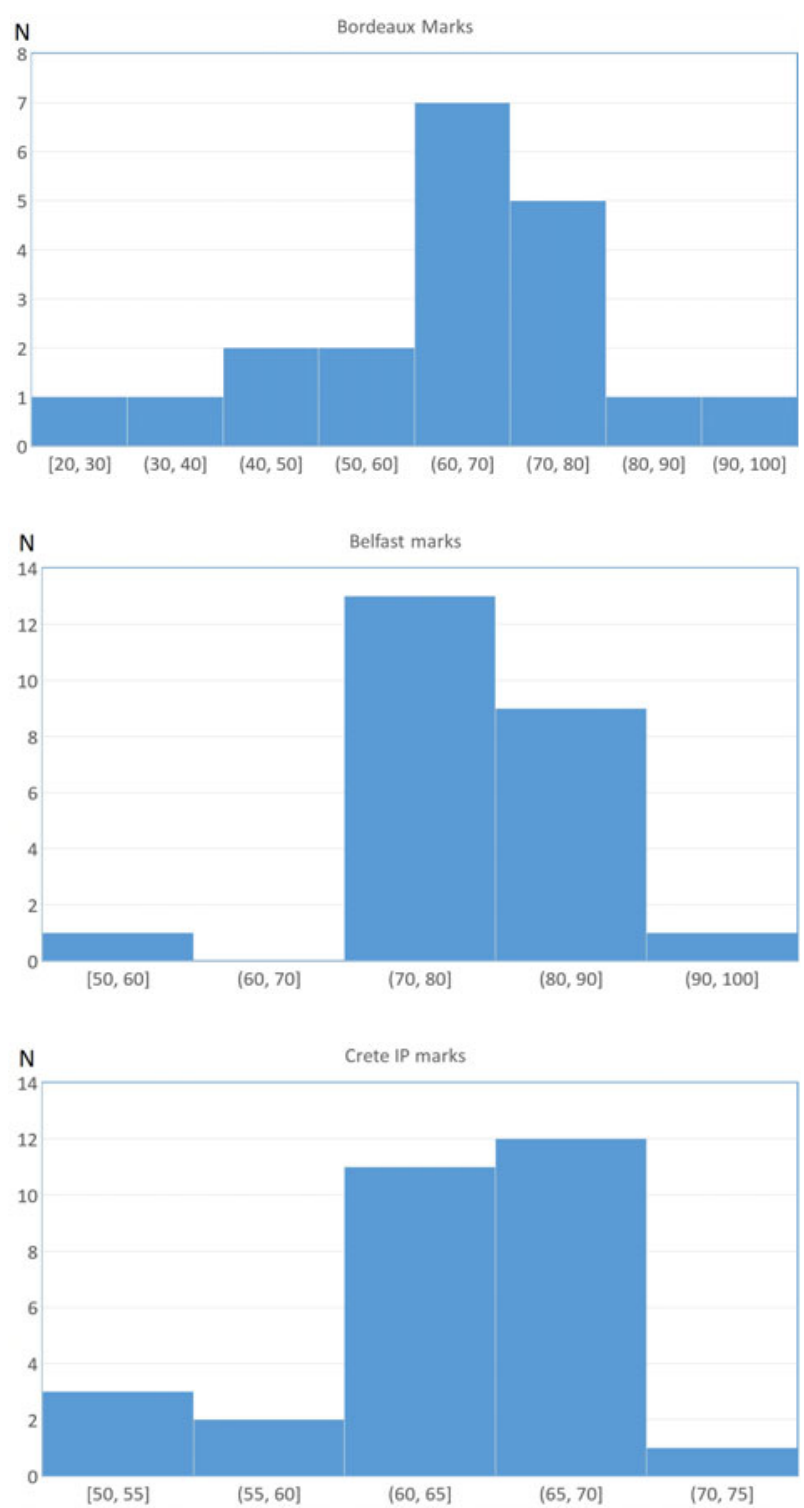

Figure 1. Marks distribution for each of the open-book examinations held at the end of each of the three LTTs (as identified in the figure) that have been held to date. It is apparent that the standard deviation about the mean varies significantly between the three events. In comparing the histograms it should be noted that the bin-width for the plot representing the Crete IP is half that of the other two plots.

will be later made accessible worldwide. The e-Class platform provides access to the digital educational and training material that has been delivered to date (or is about to be delivered).

The University of Ioannina has built and is administrating the web page of the programme ${ }^{[1]}$, in which the actions and final deliverables of each year are uploaded and further disseminated to the scientific community. The structure of the uploaded educational material allows for interactive lifelong learning and teaching. 
TEI of Crete is taking the lead in publicizing the programme using social media. This paper will also serve to disseminate some information about PowerLaPs to colleagues within the higher education sector - its production was agreed at the kick-off transnational meeting of PowerLaPs in November 2017, and finalized at the 2nd transnational meeting at Ecole Polytechnique in November 2018.

\section{Conclusions}

PowerLaPs is a novel programme of short LTTs and IPs, combining a multi-centre approach with a range of different delivery formats. To date three LTTs, including one IP, have been carried out, covering the topics of (i) plasma physics and (ii) high power laser-matter interactions and high energy density physics. All three events have been well attended with a total of 48 students having formally attended at least one of the three events. Student performance has been within the expected range and was not found to be a function of the number of LTTs attended, indicating that the academic module leads were successful in designing their modules so as to be understandable by students who were only attending one of the LTTs. The programme is now starting its second year and has three further educational LTTs (including one IP) to go before it is completed.

\section{Acknowledgements}

We acknowledge the financial support of the Erasmus Plus scheme and the IKY/Erasmus+ Hellenic National Agency. We acknowledge the support of the administrative teams of the universities involved in PowerLaPs.

\section{References}

1. PowerLaPs website: https://powerlaps.chania.teicrete.gr/.

2. Erasmus Plus website: http://ec.europa.eu/programmes/erasm us-plus/.

3. University of York Fusion MSc website: https://www.york.ac .uk/study/postgraduate-taught/courses/msc-fusion-energy/.

4. French MSc Consortium FedSPF in Plasmas and Fusion Science website: http://www.master-plasmas-fusion.fr/?lang= en.

5. Technological Educational Institute of Crete MSc in Laser Plasma and Applications website: http://www.cppl.teicrete.g r/lapla-msc-degree.

6. MSc in Physics and Technology of Thermonuclear Fusion of the Czech Technical University in Prague website: https://ww w.fjfi.cvut.cz/en/education/master-s-study/fields-of-master-sstudy/ing-physics-and-thermonuclear-fusion-technology.

7. Special Issue: Collection of Special Topic Papers on Inertial Confinement Fusion, Nuclear Fusion 54, 5 (2014).

8. National Ignition Facility website: https://lasers.llnl.gov/.

9. Extreme Light Infrastructure website: https://eli-laser.eu/.

10. CILEX-APOLLON website: https://portail.polytechnique.edu /luli/en/cilex-apollon/apollon.

11. The European X-ray Free Electron Laser website: https://ww w.xfel.eu/. 